\title{
El perdón como estrategia de afrontamiento. Una mirada desde el modelo de la complejidad del afrontamiento ${ }^{1}$
}

\author{
The forgiveness as coping. A view from the Coping Complexity Model
}

\author{
Areli Rocha ${ }^{2}$ \\ Universidad del Norte \\ María Amarís ${ }^{3}$ \\ Universidad del Norte \\ Wilson López-López ${ }^{4}$ \\ Pontificia Universidad Javeriana
}

Recepcionado (25 de Junio 2017) Aceptado (10 de Noviembre 2017)

\begin{abstract}
Resumen
El perdón se ha entendido como una estrategia de afrontamiento frente a una transgresión, de acuerdo con las propuestas del perdón basadas en la teoría del estrés y afrontamiento de Lazarus y Folkman (1986). Se llevó a cabo una revisión sistemática de la literatura para dar cuenta del desarrollo conceptual y teórico que ha tenido esta perspectiva del perdón, y se presenta el modelo de la complejidad de perdón, basado en el modelo de la complejidad de afrontamiento de Hudson (2016), como una forma de explicar el perdón y la falta de perdón considerando los aspectos que le anteceden, los procesos que involucra, los factores que influyen, y los efectos a nivel individual, interpersonal y en general resultados sociales.

Palabras claves: perdón, estrategia de afrontamiento, complejidad.
\end{abstract}

\begin{abstract}
Forgiveness has been understood as a coping strategy against a transgression, according to the proposals of forgiveness based on the theory of stress and coping of Lazarus and Folkman (1986). A systematic review of the literature was performed to give an account of the conceptual and theoretical development of this perspective of forgiveness. The forgiveness complexity model, which is based on coping complexity model of Hudson (2016), is presented as a way of explaining forgiveness and unforgiveness considering the aspects that precede it, the processes involved, the influencing factors, and the effects at the individual, interpersonal and social levels.

Key words: Forgiveness, coping, complexity.
\end{abstract}

1 Esta investigación es financiada con recursos del Fondo Nacional de Financiamiento para la Ciencia, la Tecnología e Innovación FCTeI del Sistema General de Regalías SGR, mediante la convocatoria 727 de 2015 de COLCIENCIAS.

2 Becaria doctoral de COLCIECIAS en la Universidad del Norte. E-mail: arelis.rochap0411@gmail.com

3 Doctora en Psicología, Docente Investigador de la Universidad del Norte. E-mail: mamaris@uninorte.edu.co

4 Doctor en Psicología, Docente titular de la Pontificia Universidad Javeriana. E-mail: lopezw@javeriana.edu.co 


\section{Introducción}

A lo largo de tiempo han surgido diversas definiciones del perdón, que subyacen a las distintas posturas teóricas que explican el perdón. Entre ellas se resalta el modelo cognitivoevolutivo del perdón (Enright y el Human Development Study Group, 1991), la propuesta motivacional del perdón interpersonal (McCullough, et al., 1998; Mcullough, Kurzban y Tabak, 2013), el modelo de estrés y afrontamiento del perdón (Worthington y Scherer, 2004; Strelan y Covic, 2006), entre otros.

Este artículo tiene como objetivo reconocer el desarrollo teórico del perdón como una estrategia de afrontamiento basada en un enfoque cognitivo, y proponer el modelo de la complejidad del perdón. Para tal motivo, se realizó una revisión de la literatura, mediante las base de datos $S A G E$ journals, APA PsyArticles, Academic Search Complete (EBSCO), Science Direct y Taylor \& Francis, y la base de referencias SCOPUS, con las palabras claves "forgiveness" and "coping", desde el año 2000 hasta el 2017 (agosto). Como resultado se encontró un total 204 publicaciones, de las cuales 39 artículos corresponden al área de la psicología que aborda el perdón como estrategia de afrontamiento en la línea soportada por la teoría del estrés y afrontamiento de Lazarus y Folkman (1986), y que permiten contemplar el aporte a la línea en distintos contextos (ver Tabla 1). Cabe anotar que, esta revisión excluye los trabajos que abordan el perdón dentro de las estrategias de afrontamiento religioso, para concentrarse en el perdón como estrategia de afrontamiento independiente de la religiosidad.

\section{Evolución del modelo del estrés y afrontamiento del perdón.}

La postura del perdón como estrategia de afrontamiento se fundamenta en un enfoque cognitivo y se desarrolla en base a los planteamientos de Worthington y sus colegas (Berry y Worthington, 2001; Worthington y Scherer, 2004). Worthington y Scherer (2004), basados en la teoría del estrés y afrontamiento de Lazarus y Folkman (1986), consideran que el perdón emocional corresponde a una estrategia de afrontamiento centrada en la emoción, aunque también podría ser instrumental como una estrategia centrada en el problema, y establecen cuatro proposiciones teóricas principales: 1) la falta de perdón es estresante, 2) los mecanismos de afrontamiento además del perdón pueden reducir la falta de

Tabla 1. Aportes a la comprensión del perdón como estrategia de afrontamiento en distintos contextos.

\begin{tabular}{|c|c|c|}
\hline Contexto & Aporte & Autores \\
\hline Escolar & $\begin{array}{l}\text { - Perdón como estrategia de afrontamiento frente al bullying y experiencias } \\
\text { negativas. } \\
\text { - Perdonar genera menos estados agresivos, que otras estrategias como la evitación } \\
\text { y la venganza. } \\
\text { - La evitación a corto plazo y el perdón a largo plazo son estrategias eficaces para } \\
\text { disminuir los efectos emocionales negativos del acoso escolar. } \\
\text { - Modelo para favorecer el perdón a nivel preventivo y restaurativo. }\end{array}$ & $\begin{array}{l}\text { Egan y Todorov (2009). } \\
\text { Flanagan, Vande, Ranter y Reich } \\
\text { (2012). } \\
\text { Watson, Rapee y Todorov (2016). } \\
\text { Watson, Rapee y Todorov (2017). }\end{array}$ \\
\hline Organizacional & $\begin{array}{l}\text { - Perdón para afrontar el estrés y conflictos en las relaciones laborales. } \\
\text { - Perdón como estrategia constructiva de afrontamiento frente a una injusticia en el } \\
\text { ámbito laboral. } \\
\text { - Las percepciones de la justicia organizacional facilitan el perdón entre quienes } \\
\text { tienen una orientación hacia otros. } \\
\text { - El perdón favorece la productividad. } \\
\text { - Relación del perdón con las normas y valores organizacionales. } \\
\text { - Tipos de práctica de perdón: avanzar, no tomarlo personalmente, dejar ir y perdón } \\
\text { conciliador. }\end{array}$ & $\begin{array}{l}\text { Bobocel (2013) } \\
\text { Toussaint, et al., (2016) } \\
\text { Hershcovis, Cameron, Gervais y } \\
\text { Bozeman (2017). } \\
\text { Paul, G. y Putnam, L. (2017). }\end{array}$ \\
\hline $\begin{array}{l}\text { Guerra y } \\
\text { terrorismo }\end{array}$ & $\begin{array}{l}\text { - Relación entre el perdón y los tipos de estrategias de afrontamiento. } \\
\text { - Relación entre la tendencia a perdonar y la gravedad de los síntomas de TEPT } \\
\text { entre las víctimas de ataques terroristas. } \\
\text { - Patrones de afrontamiento y su relación con el perdón. } \\
\text { - Relación del perdón no interpersonal con las categorías de respuesta al estrés: } \\
\text { control primario, control secundario, disengagement, engagement involuntario y } \\
\text { disengagement involuntario. }\end{array}$ & $\begin{array}{l}\text { Rhoades, et al. (2007) } \\
\text { Hantman (2010) } \\
\text { Weinberg, Gil y Gilbar (2015). }\end{array}$ \\
\hline
\end{tabular}




\begin{tabular}{cll}
\hline Contexto & \multicolumn{1}{c}{ Aporte } & \multicolumn{1}{c}{ Autores } \\
\hline Clínico / Salud & - Perdón como estrategia para afrontar una enfermedad crónica, como el VIH, la & Worthington, Witvliet, Pietrini y \\
& fibromialgia y la fatiga crónica. & Miller (2007) \\
& - El perdón se asocia con la calidad de vida de pacientes. & Jacinto (2009) \\
& - Perdón como estrategia de afrontamiento que reduce los síntomas del TEPT. & Swan y Scott (2009) \\
& - El perdón se asocia con menos estrés y mayor nivel de salud mental. & Ysseldyk, Matheson y Anisman \\
& - Papel mediador de los procesos de evaluación y afrontamiento en la relación & $(2009)$. \\
& entre el perdón y la depresión. & Jacinto (2010) \\
& - La relación del perdón (perdón a otro y autoperdón) con la depresión y & Sandage (2010) \\
& comportamiento suicidas. & Toussaint, et al. (2010) \\
& - El autoperdón se asocia con el afrontamiento adaptativo del duelo en cuidadores & Hirsch, Webb y Jeglic (2011) \\
& de pacientes con Alzheimer fallecidos y en familiares de una persona que se & Martin, Vosvick y Riggs (2012). \\
& suicida. & Davis, et al. (2015) \\
& - El autoperdón se relaciona con la salud física y mental. & Bac, Hyun y Ra (2015). \\
& - El perdón emocional implica cambios psicofisiológicos, y tiene consecuencias & Toussaint, Shields y Slavich (2016). \\
& directas de salud y bienestar &
\end{tabular}

\begin{tabular}{ll}
\hline Abuso / & - Perdón como estrategia para afrontar situaciones de abuso y la discriminación \\
Discriminación & racial cotidiana. \\
& - Diferencia en los efectos en la salud psicológica y las reacciones fisiológicas de la \\
& mujer abusada físicamente y la maltratada psicológicamente. \\
& - Altos niveles de perdón se asocian con menos niveles de depresión en hombres \\
& expuestos a discriminación racial. \\
& - Exploración de tres estrategias de perdón: sólo abandono de emoción negativa, \\
& sólo aumento de emoción positiva, y combinada. Existe diferencia en los \\
& resultados de depresión entre las estrategias de abandono de emoción negativa y la \\
& estrategia que no abandona las emociones negativas.
\end{tabular}

Ciclo vital

- El desarrollo del perdón en la infancia, adolescencia, adultez y vejez.

- Estilos de afrontamiento perdonador se asocian con salud mental y menos efectos del estrés vivido a lo largo de la vida.

- Las estrategias de afrontamiento adaptativas de regulación cognitiva de la emoción median la relación entre el perdón y la calidad de vida en los ancianos.
Powell, Banks y Mattis (2016) Ysseldyk, Matheson y Anisman (2017).
Allemand y Steiner (2010).

Rey y Extremera (2015).

Toussaint, Shields, Dorn y Slavich (2016)

\begin{tabular}{ll}
\hline \multicolumn{1}{c}{ Contexto } & \multicolumn{1}{c}{ Aporte } \\
\hline Contexto no & - Principios teóricos del perdón como estrategia de afrontamiento. \\
específico / & - El perdón se asocia con el afrontamiento centrado en la emoción. \\
General & - Estrategias de afrontamiento eficaces al perdonar: reinterpretación/crecimiento \\
& positivo y el afrontamiento activo. \\
& - Estrategias subyacentes al perdón. La supresión emocional disminuye las \\
& emociones negativas y la reevaluación compasiva aumenta las emociones positivas \\
& frente a un ofensor, con sus respectivos cambios fisiológicos. \\
& - Diferencias en la forma en que hombres y mujeres evalúan una situación \\
& estresante que involucra perdón. \\
& - La relación entre el perdón y la salud mental desde el modelo adaptativo continuo \\
& de la personalidad y el afrontamiento. \\
& - El papel de la brecha de injusticia en el perdón.
\end{tabular}

Medición - Instrumento de medición para identificar el perdón entre compañeros, diseñado con una conceptualización del perdón como estrategia de afrontamiento

- La medición del perdón por medio de la teoría integrada de información.

\author{
Konstam, Holmes y Levine (2003) \\ Worthington y Scherer (2004) \\ Maltby, Day y Barber (2004) \\ Strelan y Covic (2006) \\ Maltby, Macaskill y Gillett (2007). \\ Strelan y Wojtysiak (2009). \\ Witvliet, DeYoung, Hofelich y \\ DeYoung (2011). \\ Witvliet, Hofelich, Hinman y Knoll \\ (2015).
}

Davis, et al. (2016)

Jeter y Brannon (2016)

\section{Boonyarit (2017)}

López-López, W., Pineda Marín, C., Murcia León, M.C., Perilla Garzón, D.C. and Mullet, E. (2013) 


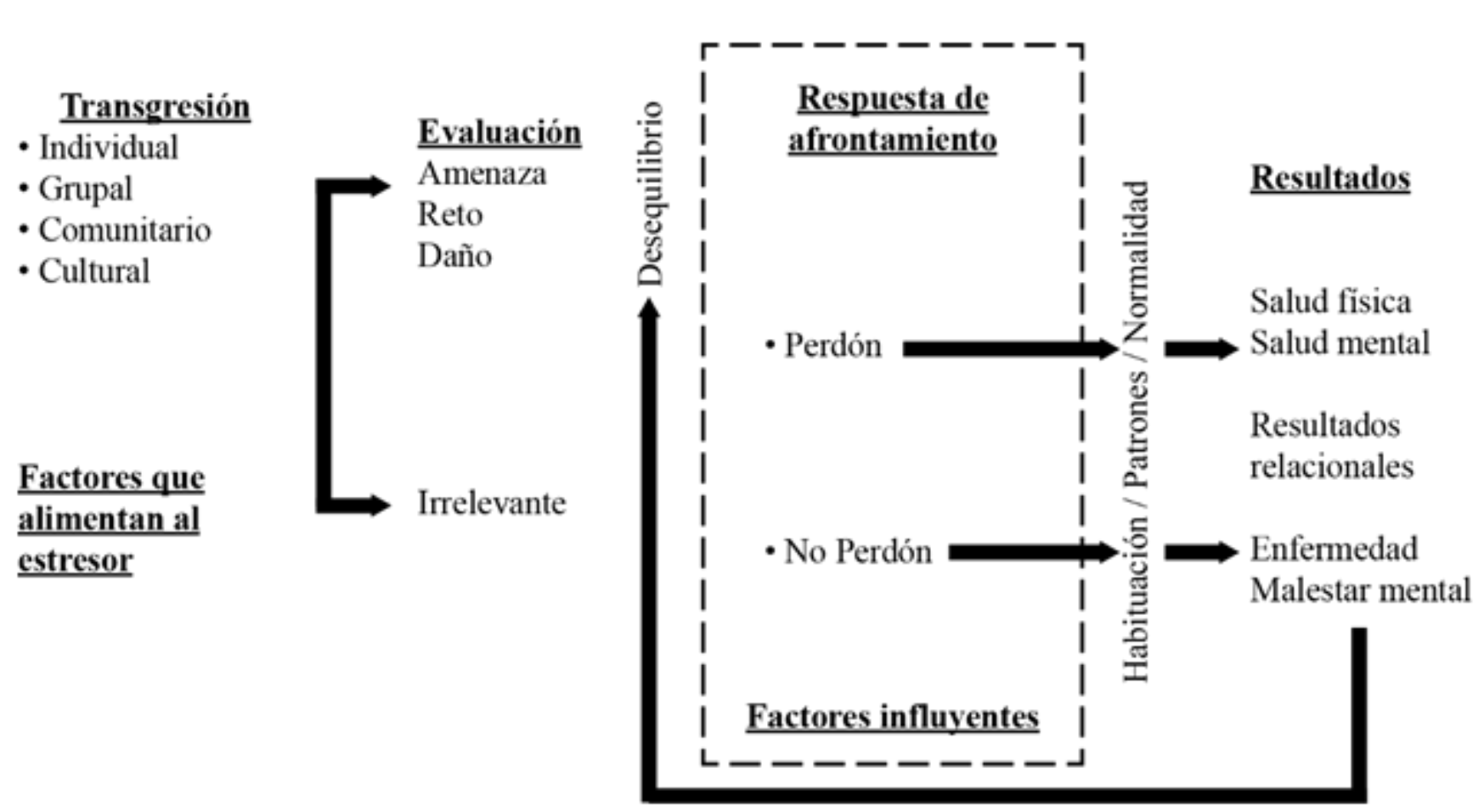

Figura 1. Modelo de la complejidad del perdón como estrategia de afrontamiento. Adaptado de Hudson (2016)

perdón, 3) el perdón reduce el estrés de la falta de perdón, y 4) el perdón como estrategia de afrontamiento está relacionado con la salud.

Posteriormente, Strelan y Covic (2006) exponen seis principios básicos: 1) el proceso del perdón es una reacción a un estresor, 2) las reacciones a una transgresión son evaluaciones primarias y secundarias, 3) las estrategias de afrontamiento describen cómo las personas perdonan, 4) el perdón y el afrontamiento también pueden orientarse hacia el futuro, 5) el perdón es un proceso intra e interpersonal, y 6) el perdón es dinámico. En base a estos postulados, algunos investigadores han aportado diferentes aspectos (ver Tabla 1), a partir de trabajos empíricos y propuestas de intervención como la de Worthington, Jennings y DiBlasio (2010).

\section{El perdón como estrategia de afrontamiento}

El perdón es considerado como una estrategia de afrontamiento que neutraliza el factor estresor originado por el trauma (Strelan y Covic, 2006), que involucra pensamientos, comportamientos, emociones y motivaciones para manejar las demandas estresantes de la falta de perdón frente a una ofensa (Worthington y Scherer, 2004), en un proceso de cambio de las respuestas negativas frente a una ofensa para disminuirlas y/o cambiarlas por respuestas positivas a nivel emocional, cognitivo y comportamental (Enright y Fitzgibbons, 2015). Tal proceso requiere tiempo (Recine, Werner y Recine, 2007), y es dinámico porque se avanza y se retrocede hasta alcanzar el punto de equilibrio psicológico (Strelan y Covic, 2006), en la medida que influyen distintos factores personales y del ambiente, como la personalidad (Maltby, et al., 2008), la espiritualidad (Davis, et al., 2013), la cultura (Ballester, Chatri, Muñoz, Rivière y Mullet, 2011), entre otros.

\section{El modelo de la complejidad del perdón}

El modelo de la complejidad de afrontamiento propuesto por Hudson (2016), busca destacar las diversas complejidades 
de las respuestas de afrontamiento. Está basado en el concepto de afrontamiento de Lazarus y Folkman (1986), en el enfoque integrativo propuesto por King y Hegadoren (2006), e incluye la teoría ecológica de Bronfenbrenner (1987). Al entender el perdón desde ese modelo, se permite aprovechar las propuestas teóricas desarrolladas, integrar la diversidad de factores que influyen en el perdón y los resultados respecto a la salud, y considerar distintos niveles que se pueden involucrar, en un esquema que incluye distintos elementos que son abordados a continuación (ver Figura 1).

\section{Transgresión.}

De acuerdo con Worthington y Scherer (2004), la teoría de Lazarus y Folkamn sugiere que la transgresión es considerada como un estresor, y las reacciones negativas frente a una transgresión pueden entenderse como reacciones de estrés. El estresor puede darse en distintos niveles (Hudson, 2016), así la transgresión puede darse a nivel intrapersonal (autoofensa), interpersonal, grupal, comunitario, e incluso a nivel cultural.

\section{Factores que alimenta al estresor.}

Existe distintos factores que alimentan una ofensa, algunos investigadores tienen en cuenta factores, tales como el contexto y momento en que sucede la ofensa (p ej. Maltby, et al., 2008; Regalia, Pelucchi, Paleari, Manzi y Brambilla, 2015) así como las características de personalidad (p. ej. Maltby, et al., 2004).

\section{Evaluación.}

La evaluación se distingue en dos aspectos valorativos: la evaluación primaria y la evaluación secundaria (Lazarus y Folkman, 1986). Una transgresión es percibida como ente estresor mediante tales evaluaciones (Worthington y Scherer, 2004). La primaria busca entender lo que es el evento y lo que significa (Taylor, 2015), y frente a cualquier transgresión puede implicar identificar que la situación ha generado un daño o pérdida, una amenaza, o un desafío (Strelan y Covic, 2006). En el caso de una ofensa a sí mismo, este proceso implica el reconocimiento de haber cometido un error (Wohl, DeShea y Wahkinney, 2008), que no necesariamente es una conducta externa sino que puede ser pensamientos, sentimientos o deseos percibidos como dañinos (Hall y Fincham, 2005). Esta evaluación también puede permitir percibir el hecho como no ofensivo, siendo en este caso una situación irrelevante para perdonar, pues "falta es la presuposición existencial del perdón” (Casullo, 2005, p. 44).

Luego surge la evaluación secundaria, que permite valorar los recursos con que se cuenta, y que tan suficientes son para satisfacer las demandas (Taylor, 2015). Frente a una ofensa, esta evaluación busca identificar qué se puede hacer, con posibles respuestas de ira, represalia o evitación si ha evaluado la ofensa como un daño, pérdida o amenaza, o con conductas conciliatorias si la concibe como un reto (Strelan y Covic, 2006). Estas evaluaciones primarias y secundarias continúan durante el proceso, así puede llegarse a la rumiación y luego replantear la transgresión, o considerar la ofensa como un desafío después de haberla considerado como daño o amenaza (Strelan y Covic, 2006).

\section{Desequilibrio.}

Como resultado de la percepción de una acción como dañina, perjudicial, inmoral o injusta se suelen generar respuestas negativas a nivel cognitivo (hostilidad, perdida de respeto al ofensor), emocional (enojo, miedo), comportamental (agresión, evitación) y motivacional (deseos de venganza) (McCulloungh y Worthington, 1999). Cabe anotar que hay diferencias en las respuestas cuando la transgresión ha ofendido a una persona distinta al ofensor, que cuando ha sido una falta contra sí misma (Prieto y Echegoyen, 2015), o cuando la ofensa ha sido contra un grupo (Enright, et al., 2016).

\section{Respuestas de afrontamiento.}

Una transgresión puede ser afrontada mediante distintas estrategias, las cuales son clasificadas por Berry y Worthignton (2001) en respuestas que perdonan y que no perdonan.

Por un lado, para Strelan y Covic (2006), la forma y el proceso en que las personas perdonan se explican mediante los dos tipos de a frontamiento propuestos por Lazarus y Folkamn (1984): 1) el afrontamiento centrado en la emoción en la medida en que se hace frente a emociones tales como la hostilidad e ira por medio de respuestas internalizadas, y 2) el afrontamiento enfocado en el problema, en cuanto se aborda el problema que causó el estrés con acciones tales como discutir con el ofensor o buscar una reparación. A diferencia del perdón a otro(s) el autoperdón sólo "es una estrategia de afrontamiento enfocada en la emoción" (Davis, et al., 2015, p. 329). 
El perdón puede darse de uno o varios niveles hacia uno $o$ varios de los niveles. Tales niveles pueden entenderse en función del objeto de perdón, así se ha estudiado el autoperdón (p ej. Wohl, et al., 2008), el perdón de uno a otro(s) o perdón interpersonal (p. ej. Enright y Human Development Study Goup, 1991), el perdón de un grupo a otro grupo o perdón intergrupal (p ej. Enright, et al., 2016), e incluso el perdón de un sujeto a una situación considerada como ofensiva ( $p$ ej. Casullo, 2005).

Por otro lado, la falta de perdón es una compleja combinación de emociones negativas hacia el ofensor, constituyéndose en una respuesta limitada, matizada y retrasada a una transgresión (Worthington y Scherer, 2004). Hay varios caminos para reducir los efectos de la falta de perdón, tales como la venganza, la justicia legal o política, la apelación a la justicia divina, la restitución, la exigencia de disculpas, el perdón, entre otros (Worthington, 2001).

\section{Factores influyentes del perdón.}

El perdón está influenciado por una serie de factores que pueden facilitar o limitar la práctica del perdón, por lo que tales factores también se constituyen en factores que influyen en la falta de perdón. En la tipología tripartita del perdón se encuentran categorizados los factores asociados al perdón interpersonal (Fehr, Gelfand y Nag, 2010), e intergrupal (Van Tongeren, Burnette, O'Boyle, Worthington y Forsyth, 2014), además de factores como el tiempo, la edad, y el género. Por ejemplo, Rey y Extremera (2016) resaltan las diferencias entre hombres y mujeres que existen al momento de afrontar una transgresión.

Además, algunos de los factores influyentes son recursos de afrontamiento, que son factores que preceden e influyen en el afrontamiento y en el estrés (Lazarus y Folkman, 1986). Se han estudiado algunos recursos asociados al perdón y el estrés por una transgresión, tales como la autoestima (Strelan y Zdaniuk, 2015), el autoconcepto (Van Dyke y Elias, 2008), las creencias sobre el mundo (Strelan y Sutton, 2011; Bartholomaeus y Strelan, 2016), el nivel de ingresos económicos (Konstam, et al., 2003), entre otros.

\section{Habituación y Patrones.}

La habituación "se refiere a la pérdida de respuesta de un organismo frente a un estímulo repetido o constantemente presente" (Greenwood, 1993, citado por Hudson, 2016). Esta disminución de la respuesta de estrés debido a la repetición puede resultar del afrontamiento (Lazarus y Folkman, 1984). Por eso, la persona expuesta a una constante de ofensas puede habituarse disminuyendo las respuestas de estrés en la medida que aprende a afrontarlas. El estudio de Toussaint, et al. (2016) concluye que el estilo de afrontamiento perdonador puede minimizar los efectos del estrés al que se ha estado expuesto a lo largo de la vida.

El patrón tiene que ver con la recurrencia de respuesta de afrontamiento, la cual puede evoluacionar con el tiempo debido a la necesidad de responder a situaciones ofensivas. Frente a distintas oensas, el individuo con el correr del tiempo puede adquirir y/o cambiar el patrón de respuesta, dependiendo de algunos factores, como el desarrollo (Enright, 1994), los rasgos de la personalidad (Lawler-Row y Pifri, 2006), y la cultura (Enright, et al., 2016).

\section{Normalidad.}

La normalidad es un estado devuelto tan cerca de donde estaba la persona antes del estresor (Hudson, 2016). De ese modo, el perdón permite recuperar el equilibrio (Strelan y Covic, 2006), posibilitando eliminar o disminuir las distorsiones generados por el trauma (Finch, 2006).

\section{Resultados}

Karremans y Van Lange (2008) identifican tres niveles en los que operar las consecuencias del perdón:

1. A nivel intrapersonal, se sugieren los resultados en temas de salud física y mental (Karremans y Van Lange, 2008). Por un lado, la falta de perdón conduce a respuestas que involucran componentes atencionales, fisiológicos y comportamentales que causan ansiedad, depresión, hostilidad y hasta enfermedades cardiacas, y por otro lado, el perdón se asocia con resultados positivos y factores que favorecen el bienestar psicológico y la salud física, en la medida que se asocia con la reducción del afecto negativo, con un sistema inmunológico más fuerte, menos reactividad fisiológica al estrés, entre otros aspectos (Lavelock, et al., 2015). En caso del autoperdón, practicarlo con facilidad no siempre es una alternativa saludable, pues podría aliviar la culpa y la vergüenza facilitando al individuo reincidir en la conducta ofensiva, como las adicciones, y así podría perpetuar ciclos de conductas destructivas, debilitando los resultados de salud física y mental (Davis, et al., 2015).

2. A nivel interpersonal, se resalta el bienestar y la persistencia de las relaciones (Karremans y Van Lange, 
2008). El perdón puede posibilitar la reconciliación, aunque no siempre es una consecuencia positiva del perdón, porque puede poner al ofendido en una situación de riesgo de ser revictimizado si se trata de una relación abusiva y maltratadora, por lo tanto es pertinente tener en cuenta el marco relacional (Guzmán, 2010). Sin embargo, el perdón a sí mismo es un proceso que si involucra la reconciliación, porque el ofendido y el ofensor son la misma persona (Hall y Fincham, 2005).

3. A nivel general, se sugiere que los efectos del perdón pueden llegar a transcender al individuo y su relación con el ofensor, en la medida que el estado psicológico del perdón puede motivar comportamientos prosociales hacia los demás (Karremans y Van Lange, 2008). Así, el perdón puede (o no) producir perspectivas más pacíficas y armoniosas, (Worthington, 2005), ser una alternativa creativa para la transformación de las violencias para la construcción de paz (López-López, Pineda Marín, Murcia León, Perilla Garzón, and Mullet, 2013; López, Pineda y Mullet, 2014; Villa, 2016 : Cortez, Torres, López-López, Perez, Pinedad Marin, 2016; Mullet, Lopez-Lopez, Kpanake, Armange, Neto, Kamble, Muñoz, Sorum, Mukashema, Guedez, 2016; López-López, Andrade, y Correa, 2016), y cumplir un papel importante en la curación de daños a nivel de grupo (Strelan y Covic, 2006), en el mantenimiento de la red de apoyo social, y el incremento de factores relacionales asociados a las habilidades sociales, calidad en las relaciones y manejo de conflictos (Lavelock, et al., 2015).

\section{Discusión y conclusión}

La revisión de la literatura realizada da cuenta de diversos aportes teóricos y empíricos que posibilitan la comprensión del perdón como estrategia de afrontamiento, mostrando el auge emergente de estudios en diversos contextos, abriendo posibilidades para futuras investigaciones, proveyendo elementos aplicables a procesos de intervención, y proporcionando un panorama que precede al modelo de la complejidad del perdón.

Este modelo amplía la perspectiva ya desarrollada del modelo de estrés y afrontamiento aplicado al perdón, en cuanto lo articula con las propuestas teóricas de los sistemas y de la complejidad, dando cuenta de: a) la funcionalidad del perdón frente a una transgresión, que puede verse en distintos niveles de acción y asociarse a una compleja serie de factores que influyen en el proceso de afrontamiento, $b$ ) su operatividad, en la medida que se presenta un esquema del recorrido desde la ofensa hasta los resultados del afrontamiento, contemplando los procesos que operan en el transcurso, y c) su naturaleza entendida como una estrategia de afrontamiento, sin entrar en contradicción con otras propuestas, como la naturaleza moral del perdón explicada desde el modelo cognitivo-evolutivo, que ubica al perdón dentro de las virtudes morales pero asumiendo que tal virtud puede aparecer como una estrategia de afrontamiento (Enright y Fitzgibbons, 2015).

La articulación del modelo con los sistemas muestra oportunidades de investigación. Osman y Miranda (2016) desarrollaron una escala que permite identificar diferencias individuales del perdón de rasgos en seis niveles sistémicos, pero también es necesario indagar el perdón episódico en uno de los sistemas relacionado con los demás sistemas. Además, con el fin de determinar los factores que operan en cierto nivel y su relación con los demás niveles, vale la pena continuar con los esfuerzos de clasificación que han hecho algunos autores (Palanski, 2012; Fehr, et al, 2010; Van Tongeren, et al, 2014), de identificación mediante estudios, y de construcción de instrumento de medición de los factores relacionados al perdón en distintos niveles, como el inventario desarrollado de Blatt y Wertheim (2015) referente al perdón interpersonal. También, se hace pertinente profundizar en la relación de los factores que alimentan el evento estresor con el desequilibrio, el proceso del perdón y la salud, así como precisar los recursos de afrontamiento que cumplen un papel en el perdón.

Por otro lado, adicionado al modelo de Hudson (2016), este modelo resalta la estructura comprendida por antecedentes, procesos y consecuencias, acorde con el modelo de estrés y afrontamiento que presentan Lazarus y Folkman (1986). Esta distinción invita a considerar al perdón teniendo en cuenta: a) los procesos y elementos que le anteceden, los cuales pueden abonar a la comprensión del fenómeno, b) los resultados del perdón para enriquecer el análisis de los efectos del afrontamiento como indicadores de perdón, y c) los antecedentes del perdón en conjunto con los efectos, para dar cuenta con mayor precisión del impacto del perdón en la reducción del daño causado y los beneficios en la salud y las relaciones sociales, y además aportar a la comprensión de la función mediadora del perdón en la relación entre el trauma y la salud mental, como lo han hecho Toussaint, et al., (2016).

El modelo, dentro de las respuestas de afrontamiento aborda al perdón y la falta de perdón, sin embargo no se especifican otras estrategias como la búsqueda de justicia legal o política, la evitación, la apelación a la justicia divina, 
la restitución, entre otros (Worthington, 2001; Worthington y Scherer, 2004), que pueden ser tenidos en cuenta en futuras contribuciones al modelo, para ampliar la visión respecto a las distintas formas de afrontar una ofensa y las estrategias para afrontar la falta de perdón sin perdonar. Así mismo, vale la pena continuar matizando las diferencias, relaciones y consecuencias de los distintos tipos y estilos de afrontamiento al perdonar, considerando por un lado al perdón centrado en la emoción y el perdón centrado en el problema, por otro lado, la disminución de los aspectos negativos, el aumento de aspectos positivos y la combinación de ambos, y también el perdón a corto y largo plazo.

También, se requiere hacer estudios experimentales que identifiquen el estado del individuo antes del evento ofensivo para compararlo con el estado posterior al perdón, y así confirmar la normalidad, continuar haciendo estudios que den cuenta de la habituación frente a la exposición prolongada de ofensas y a lo largo de la vida, y contribuir a la identificación de distintos aspectos que moldean el patrón de respuesta en función del perdón, como la educación por parte de los padres y figuras de autoridad (Worthington, et al., 2010), ya que el entorno familia representa un espacio socioeducativo para el modelamiento de estrategias de afrontamiento individual (Amarís, Madariaga, Valle y Zambrano, 2013).

Por último, se hace hincapié en la relación perdón-salud. Distintos estudios han ofrecido una relación unidireccional, donde se muestra los beneficios en la salud física y mental al perdonar, y sus consecuencias negativas asociadas a la falta de perdón. Sin embargo, algunos autores sugieren esta relación en un sentido bidireccional, donde el perdón influye en la salud y la salud influyen en el perdón (Lavelock, et al., 2015; Bono, McCullough y Root, 2008). El modelo de la complejidad del perdón, soporta la relación bidireccional al considerar que la salud es un recurso individual en el afrontamiento (Lazarus y Folkman, 1986), pues es un estado del ofendido y por ende es un factor que precede y a su vez influyen en los procesos que asuma el sujeto, además de ser considerada por Egan y Todorov (2009) como un amortiguador de los efectos emocionales de las transgresiones. Lo anterior deja en evidencia la relación influyente de la salud sobre el perdón, que añadido a la evidencia empírica de los efectos positivos del perdón sobre la salud, se plantea una relación bidireccional de mutua dependencia que vale la pena esclarecer en futuras investigaciones.

\section{Referencias Bibliográficas}

Allemand, M. y Steiner, M. (2010). Verzeihen und Selbstverzeihen über die Lebensspanne Gegenwärtiger Forschungsstand und Forschungsperspektiven. Zeitschrift fur Entwicklungspsychologie und Padagogische Psychologie, 42, 63-78. doi: 10.1026/0049-8637/a000012

Amarís, M., Madariaga, C., Valle, M. y Zambrano, J. (2013). Estrategias de afrontamiento individual y familiar frente a situaciones de estrés psicológico. Revista Psicología desde el Caribe, 30, 123-145.

Bae, S., Hyun, M. y Ra, Y. (2015). Mediating effects of forgiveness and emotion-focused coping on post-traumatic stress disorder symptoms caused by physical injury and perceived threat. Asia-Pacific Psychiatry. 7, 164-172. doi:10.1111/appy.12142

Ballester, S.; Chatri, F.; Muñoz, M.; Rivière, S. y Mullet, E. (2011). Forgiveness-related motives: A structural and cross-cultural approach. Social Science Information, 50, 178-200. doi: 10.1177/0539018411398418

Bartholomaeus, J. y Strelan, P. (2016). Just world beliefs and forgiveness: Themediating role of implicit theories of relationships. Personality and Individual Differences, 96, 106-110. doi: 10.1016/j.paid.2016.02.081

Berry, M. y Worthingtin, E. Jr. (2001). Forgivingness, Relationship Quality, Stress While Imagining Relationship Events, and Physical and Mental Health. Journal of Counseling Psychology, 48, 447-755. doi: 10.1037//0022-0167.48.4.447

Blatt, L. y Wertheim, E. (2015). Development of the Factors Related to Forgiveness Inventory (FRFI). Assessing social-cognitive facilitators and inhibitors of interpersonal forgiveness. European Journal of Psychological Assessment, 31, 100-108. doi: 10.1027/1015-5759/a000211

Bobocel, D. R. (2013). Coping with unfair events constructively or destructively: The effects of overall justice and self-other orientation. Journal of Applied Psychology, 98, 720-731. doi: 10.1037/a0032857

Bono, G., McCullough, M. y Root, L. (2008). Forgiveness, Feeling Connected to Others, and Well-Being: Two Longitudinal Studies. Personality and social psychology bulletin, 34, 182-195. doi: 10.1177/0146167207310025

Boonyarit, I. (2017). Assessing forgiveness in interpersonal conflict among thai emerging adults: The peer forgiveness scale. International Journal of Behavioral Science, 12, 1-18.

Bronfenbrenner, U. (1987). La ecología del desarrollo humano. Experimentos en entornos naturales y diseñados. Barcelona: Paidós.

Casullo, M. (2005). La capacidad para perdonar desde una perspectiva psicológica. Revista de Psicología de la PUCP, 23, pp. 40-63. Recuperado de: http://www.scielo.cl/pdf/psykhe/v19n1/art02.pdf

Cortez, A., Torres, A. Lopez-Lopez, W., Perez, C. Pinedad Marin. (2016) Comprensiones sobre el perdón y la reconciliación en el contexto del conflicto armado colombiano. Psychosocial intervention. 19-25. Vol. 25 No.1 DOI: 10.1016/j.psi.2015.09.004

Davis, D. E.; Ho, M. Y.; Griffin, B. J; Bell, C.; Hook, J. N.; Van Tongeren, D. R.; DeBlaere, C.; Worthignton, E. L. y Wetbrook, C. J. (2015). Forgiving the self and physical and mental health correlates: A me- 
ta-analytic review. Journal of Counseling Psychology. 62, 329-335. doi: $10.1037 /$ cou0000063

Davis, D. E.; Worthington, E. L. Jr.; Hook, J. N. y Hill, P. C. (2013). Research on religion/spirituality and forgiveness: A meta-analytic review. Psychology of Religion and Spitituality. 5, 233-241. doi: 10.1037/a0033637

Davis, D., Yang, X., DeBlaere, C., McElroy, S., Van Tongeren, D., Hook, J. y Worthington, E. (2016). The injustice gap. Psychology of Religion and Spirituality, 8, 175-184. doi: 10.1037/rel0000042

Egan, L., y Todorov, N. (2009). Forgiveness as a coping strategy to allow school students to deal with the effects of being bullied: Theoretical and empirical discussion. Journal of Social and Clinical Psychology, 28, 198-222. doi: 10.1521/jscp.2009.28.2.198

Enright, R. (1994). Piaget on the moral development of forgiveness: Identity o reciprocity?. Human Development, 37, 63-80. doi: 10.1159/000278239

Enright, R. y The Human Development Study Group. (1991). The moral development of forgiveness. In W. Kurtines y J. Gewirtz (Eds.), Handbook of moral behavior and development. Vol. 1 (pp. 123-152). Hillsdale, NJ: Erlbaum.

Enright, R. y Fitzgibbons, R. (2015). Forgiveness Therapy: An Empirical Guide for Resolving Anger and Restoring Hope. E.U.: American Psychological Association.

Enright, R.; Lee, Y.; Hirshberg, M.; Litts, B.; Schirmer, E.; Irwin, A.; Klatt, J.; Hunt, J. y Yu Song, J. (2016). Examining Group Forgiveness: Conceptual and Empirical Issues. Peace and Conflict: Journal of Peace Psychology, 22, 153-162. doi: 10.1037/pac0000153

Fehr, R., Gelfand, M. J. y Nag, M. (2010). The road to forgiveness: A meta-analytic synthesis of its situational and dispositional correlates. Psychological Bulletin, 136, 894-914. doi: 10.1037/a0019993

Flanagan, K., Vande, K., Ranter, J. y Reich, H. (2012). The potential of forgiveness as a response for coping with negative peer experiences. Journal of Adolescence, 35, 1215-1223. doi:10.1016/j.adolescence.2012.04.004

Guzmán, M. (2010). El perdón en relaciones cercanas: Conceptualización desde una perspectiva psicológica e implicancias para la práctica clínica. Psykhe, 19, 19-30. Recuperado de: http://www.scielo.cl/pdf/ psykhe/v19n1/art02.pdf

Hall, J. H. y Fincham, F. D. (2005). Self-forgiveness: The stepchild of forgiveness research. Journal of Social and Clinical Psychology, 24, 621-637. doi: 10.1521/jscp.2005.24.5.621

Hantman, S. (2010). Holocaust survivor typology and forgiveness. Journal of Human Behavior in the Social Environment, 20, 507-524. doi: 10.1080/10911350903275226

Hershcovis, M. S., Cameron, A.-F., Gervais, L. y Bozeman, J. (2017). The effects of confrontation and avoidance coping in response to workplace incivility. Journal of Occupational Health Psychology, Advance online publication. doi: 10.1037/ocp0000078

Hirsch, J.K., Webb, J.R. y Jeglic, E.L. (2011). Forgiveness, depression, and suicidal behavior among a diverse sample of college students. Journal of Clinical Psychology, 67, 896-906. doi: 10.1002/jclp.20812
Hudson, K. (2016). Coping complexity model: coping stressors, coping influencing factors, and coping responses. Psychology, 7, 300-309. doi: $10.4236 /$ psych.2016.73033

Jacinto, G. (2009) The self-forgiveness process of caregivers after the death of care-receivers diagnosed with Alzheimer's disease. Journal of Social Service Research, 36, 24-36. doi: 10.1080/01488370903333538

Jacinto, G. (2010). Caregivers' Coping and Self-Forgiveness After the Death of a Care-Receiver. Journal of Social Service Research, 36, 206-215. doi: 10.1080/01488371003697939

Jeter, W. K. y Brannon, L. A. (2016). Perceived and actual effectiveness of coping strategies used when forgiving. Counseling and Values 61, 176-191. doi: 10.1002/cvj.12036

Karremans, J. y Van Lange, P. (2008). Forgiveness in personal relationships: Its malleability and powerful consequences. European Review of Social Psychology, 19, 202-241. doi: 10.1080/10463280802402609

King, S. L., y Hegadoren, K. M. (2006). An Integrative Science Approach: Value Added in Stress Research. Nursing and Health Sciences, 8, 114119. doi: 10.1111/j.1442-2018.2006.00256.x

Konstam, V.; Holmes, W. y Levine, B. (2003). Empathy, Selfism, and Coping as Elements of the Psychology of Forgiveness: A Preliminary Study. Counseling and values, 47, 172-183. doi: 10.1002/j.2161007X.2003.tb00264.X

Lavelock, C., Snipes, D., Griffin, B., Worthington, E., Davis, D., Hook, J., Benotsch, E. y Ritter, J. (2015). Chapter 3: A conceptual model of forgiveness and healt. En: Toussaint, L., Worthington, E: y Williams, D. (Eds.). Forgiveness and Health. Scientific evidence and theories relating forgiveness to better health (pp. 29-42). Nueva York: Springer.

Lawler-Row, K. y Piferi, R. (2006). The forgiving personality: Describing a life well lived? Personality and Individual Differences, 41, 1009-1020. doi:10.1016/j.paid.2006.04.007

Lazarus, R. y Folkman, S. (1986). Estrés y procesos cognitivos. Barcelona, España: Ediciones Martínez Roca, S. A.

López-López,W., Pineda, C. y Mullet, E. (2014). El perdón como proceso de transformación para la paz. En: Ruiz, J. (Ed.). Psicología Social y Justicia (pp. 225-238). Bogotá, D.C.: Universidad Nacional de Colombia Editorial.

López-López, W., Pineda Marín, C., Murcia León, M.C., Perilla Garzón, D.C. and Mullet, E. (2013). Forgiving Perpetrators of Violence: Colombian People's Positions. Social Indicators Research, 114, 287-301. doi: 10.1007/s11205-012-0146-1. (ISI-SCOPUS)

López-López, W., Andrade, A. y Correa, A. (2016) El proceso de pedir perdón como condición necesaria para la construcción de paz en medio del conflicto armado en Colombia. Revista Argentina de Clínica Psicológica Vol. XXV, N², 187-194.

Maltby, J., Day, L. y Barber, L. (2004). Forgiveness and mental health variables: Interpreting the relationship using an adaptational-continuum model of personality and coping. Personality and Individual Differences, 37, 1629-1641. doi:10.1016/j.paid.2004.02.017 
Maltby, J., Macaskill, A. y Gillett, R. (2007). The cognitive nature of forgiveness: Using cognitive strategies of primary appraisal and coping to describe the process of forgiving. Journal of Clinical Psychology, 63, 555-566. doi: 10.1002/jclp.20367

Maltby, J.; Wood, A.; Day, L.; Kon, T., Colley, A. y Linley, A. (2008). Personality predictors of levels of forgiveness two and a half years after the transgression. Journal of Research in Personality, 42, 1088-1094. doi: 10.1016/j.jrp.2007.12.008

Martin, L.A., Vosvick, M. y Riggs, S.A. (2012). Attachment, forgiveness, and physical health quality of life in HIV + adults. AIDS Care - Psychological and Socio-Medical Aspects of AIDS/HIV, 24, 1333-1340. doi: 10.1080/09540121.2011.648598

McCullough, M. E., Kurzban, R., y Tabak, B. A. (2013). Cognitive systems for revenge and forgiveness (with commentaries and response). Behavioral and Brain Sciences, 36, 1-58. doi: 10.1017/S0140525X11002160

McCullough, M. E., Rachal, K. C., Sandage, S. J., Worthington, E. L., Brown, S. W., y Hight, T. L. (1998). Interpersonal forgiving in close relationships. II: Theoretical elaboration and measurement. Journal of Personality and Social Psychology, 75, 1586-1603.

McCulloungh, M. y Worthington, E. Jr. (1999). Religion and the forgiving personality. Journal of Personality, 67, 1141-1164. doi: 10.1111/14676494.00085

Mullet, E., Lopez-Lopez,W., Kpanake, L.; Armange, R; Neto, F; Kamble, S; Muñoz Sastre, M T; Sorum, P C; Mukashema, I; Guedez, A G; (2016). Functional measurement in the field of Ethics in Politics. Universitas Psychologica, 15(3) 292-344. doi:10.11144/Javeriana.upsy15-3.fmf.

Osman, M. y Miranda, D. (2014). Validation of a new measure of trait forgiveness: Multi-Systemic Forgiveness Scale (MSFS). doi: 10.1016/j. paid.2013.07.344

Palanski, M. E. (2012). Forgiveness and Reconciliation in the Workplace: A Multi-Level Perspective and Research Agenda. J Bus Ethics, 109, 275-287. doi: 10.1007/s10551-011-1125-1

Paul, G. y Putnam, L. (2017). Moral Founddations of forgiving in the workplace. Western Journal of Communication, 81, 43-63. doi: 10.1080/10570314.2016.1229499

Powell, W., Banks, K. H., y Mattis, J. S. (2016). Buried hatchets, marked locations: forgiveness, everyday racial discrimination, and African American men's depressive symptomatology. American Journal of Orthopsychiatry. Advance online publication. doi: 10.1037/ort0000210

Prieto, M. y Echegoyen, I. (2015). ¿Perdón a uno mismo, autoaceptación o restauración interpersonal? Cuestiones abiertas en psicología del perdón. Papeles del Psicólogo, 36, 230-237. Recuperado de: http:// www.redalyc.org/pdf/778/77842122009.pdf

Recine, A. G.; Werner, J. S. y Recine, L. (2007). Concept analysis of forgiveness with a multi-cultural emphasis. Journal of Advanced Nursing 59, 308-316 doi: 10.1111/j.1365-2648.2007.04343.

Regalia, C.; Pelucchi, S.; Paleari, F.; Manzi, C. y Brambilla, M. (2015).
Forgiving the terrorists of the Years of Lead in Italy: The role of restorative justice beliefs and sociocognitive determinants. Group Process \& Intergroup relations, 18, 609-623. doi: 0.1177/1368430215570503

Rey, L. y Extremera, N. (2015). Forgiveness and health-related quality of life in older people: Adaptive cognitive emotion regulation strategies as mediators. Journal of Health Psychology, 21, 2944-2954. doi: $10.1177 / 1359105315589393$

Rey, L. y Extremera, N. (2016). Agreeableness and interpersonal forgiveness in young adults: the moderating role of gender. Terapia Psicológica, $34,103-110$.

Rhoades, G.K., McIntosh, D.N., Wadsworth, M.E., Ahlkvist, J., Burwell, R., Gudmundsen, G., Raviv, T. y Rea, J.G. (2007). Forgiving the September 11th terrorists: Associations with coping, psychological distress, and religiosity. Anxiety, Stress and Coping, 20, 109-128. doi: 10.1080/10615800701195405

Sandage, S. (2010). Intergenerational suicide and family dynamics: A hermeneutic phenomenological case study. Contemporary Family Therapy: An International Journal, 32, 209-227. doi:10.1007/s10591-009-9102-x

Strelan, P. y Covic, T. (2006). A review of forgiveness process models and a coping framework to guide future research. Journal of Social \& Clinical Psychology, 25, 1059-1085. doi: 0.1521/jscp.2006.25.10.1059

Strelan, P. y Sutton, R. (2011). When just-world beliefs promote and when they inhibit forgiveness. Personality and Individual Differences, 50, 163-168. doi: 10.1016/j.paid.2010.09.019

Strelan, P. y Wojtysiak, N. (2009). Strategies for coping with interpersonal hurt: Preliminary evidence for the relationship between coping and forgiveness. Counseling and Values, 53, 97-111. doi: 10.1002/j.2161007X.2009.tb00117.x

Strelan, S y Zdaniuk, A(2015). Threatened State Self-Esteem Reduces Forgiveness. Self and Identity, 14, 16-32, DOI: 10.1080/15298868.2014.889034

Swan, A. y Scott, C. (2009). Complicated grief: implications for the treatment of post-traumatic stress disorder in couples. Sexual \& Relationship Therapy, 24, 16-29. doi: 10.1080/14681990802641299

Taylor, S. (2015). Health Psychology. Nueva York: McGraw Hill Education. Toussaint, L., Overvold-Ronningen, M., Vincent, A., Luedtke, C., Whipple, M., Schriever, T. y Luskin, F. (2010). Implications of forgiveness enhancement in patients with fibromyalgia and chronic fatigue syndrome. Journal of Health Care Chaplaincy, 16, 123-139. doi: 10.1080/08854726.2010.492713

Toussaint, L., Shields, G., Dorn, G. y Slavich, G. (2016). Effects of lifetime stress exposure on mental and physical health in young adulthood: How stress degrades and forgiveness protects health. Journal of Health Psychology, 21, 1004-1014. doi: 10.1177/1359105314544132

Toussaint, L., Worthingtin, E., Van Tongeren, D., Hook, J., Berry, J., Miller, A. y Davis, D. (2016). Forgiveness Working. Forgiveness, health, and productivity in the workplace. American Journal of Health Promotion, 1-9. doi: $10.1177 / 0890117116662312$ 
Toussaint, L. L., Shields, G. S. y Slavich, G. M. (2016). Forgiveness, stress, and health: a 5-week dynamic parallel process study. Annals of Behavioral Medicine 50, 727-735. doi: 10.1007/s12160-016-9796-6

Van Tongeren, D., Burnette, J., O’Boyle, E., Worthington, E. y Forsyth, D. (2014) A meta-analysis of intergroup forgiveness, The Journal of Positive Psychology, 9, 81-95. doi: 10.1080/17439760.2013.844268

Van Dyke, C. y Elias, M. (2008). How Expressions of Forgiveness, Purpose, and Religiosity Relate to Emotional Intelligence and Self-Concept in Urban Fifth-Grade Students. American Journal of Orthopsychiatry, 78, 481-493. doi: 10.1037/a0014451

Villa, J. D. (2016). Perdón y reconciliación: una perspectiva psicosocial desde la noviolencia. Polis, Revista latinoamericana, 15, 131-157. Recuperado de: http://polis.revues.org/11553

Watson, H., Rapee, R. y Todorov, N. (2017). Forgiveness Reduces Anger in a School Bullying Context. Journal of Interpersonal Violence, 32, 1642-1657. doi: 10.1177/0886260515589931

Watson, H., Rapee, R. y Todorov, N. (2016). Imagery rescripting of revenge, avoidance, and forgiveness for past bullying experiences in young adults. Cognitive Behaviour Therapy, 45, 73-89. doi: 10.1080/16506073.2015.1108360

Weinberg, M., Gil, S. y Gilbar, O. (2015). Forgiveness, coping, and terrorism: Do tendency to forgive and coping strategies associate with the level of posttraumatic symptoms of injured victims of terror attacks? Journal of Clinical Psychology, 70, 693-703. doi: 10.1002/jclp.22056

Witvliet, C., Hofelich, A., Hinman, N. y Knoll, R. (2015). Transforming or restraining rumination: The impact of compassionate reappraisal versus emotion suppression on empathy, forgiveness, and affective psychophysiology. The journal of Positive Psychology, 10, 248-261. doi: 10.1080/17439760.2014.941381

Witvliet, C.; DeYoung, N.; Hofelich, A. y DeYoung P. (2011). Compassionate reappraisal and emotion suppression as alternatives to offense-focused rumination: Implications for forgiveness and psychophysiological well-being. The Journal of Positive Psychology, 6, 286-299. doi: 10.1080/17439760.2011.577091

Wohl, M.; DeShea, L. y Wahkinney, R. (2008). Looking Within: Measuring State Self-Forgiveness and Its Relationship to Psychological Well-Being. Canadian Journal of Behavioural Science, 40, 1-10. doi: 10.1037/0008-400x.40.1.1.1

Worthington, E. (2001). Unforgiveness, forgiveness, and reconciliation in societies. En: R. G. Helmick y R. L. Petersen (Eds.), Forgiveness and Reconciliation: Religion, Public Policy, and Conflict Transformation (pp. 161-182). Philadelphia: Templeton Foundation Press.

Worthington, E. (2005). Initial questions about the art and sciencie of forgiving. En: Worthington, E. Jr. (Ed.). Handbook of forgiveness. New York: Taylor \& Francis Group, LLC.

Worthington, E. Jennings, D. y DiBlasio, F. (2010). Interventions to promote forgiveness in couple and family context: conceptualization, review, and analysis. Journal of Psychology and Theology, 38, 231-245.

Worthington E., Witvliet, C., Pietrini, P. y Miller, A. (2007). Forgiveness, health, and well-being: A review of evidence for emotional versus decisional forgiveness, dispositional forgivingness, and reduced unforgiveness. Journal of Behavioral Medicine, 30, 291-302. doi: 10.1007/ s10865-007-9105-8

Worthington, E. y Scherer, M. (2004). Forgiveness is an emotion-focused coping strategy that can reduce health risks and promote health resilience: theory, review, and hypotheses. Psychology \& Health, 19, 385-405. doi: 10.1080/0887044042000196674

Ysseldyk, R., Matheson, K. y Anisman, H. (2009). Forgiveness and the appraisal-coping process in response to relationschip conflicts: Implications for depressive symptoms. Stres, The International Journal on the Biology of Stress, 12, 152-166. doi: 10.1080/10253890802228178

Ysseldyk, R., Matheson, K. y Anisman, H. (2017). Revenge is sour, but is forgiveness sweet? Psychological health and cortisol reactivity among women with experiences of abuse. Journal of Health Psychology, 0, 1-19. doi: $10.1177 / 1359105317714319$ 
